Ann. Funct. Anal. 6 (2015), no. 2, 49-59

http://doi.org/10.15352/afa/06-2-5

ISSN: 2008-8752 (electronic)

http://projecteuclid.org/afa

\title{
INTERPLAY OF WIENER-HOPF AND HANKEL OPERATORS WITH ALMOST PERIODIC FOURIER SYMBOLS ON STANDARD AND VARIABLE EXPONENT LEBESGUE SPACES
}

\author{
L. P. CASTRO* AND A. S. SILVA \\ Communicated by C. P. Niculescu
}

\begin{abstract}
Wiener-Hopf plus Hankel and Wiener-Hopf minus Hankel operators in both frameworks of standard and variable exponent Lebesgue spaces are considered in this paper. The main aim is to describe certain dependencies between the Fredholm property of some Wiener-Hopf operators acting between variable exponent Lebesgue spaces and the invertibility of Wiener-Hopf plus and minus Hankel operators on all the standard Lebesgue spaces. Different types of Fourier symbols will be used but special focus will be considered on the Wiener subclass of almost periodic matrix functions. In the first part of the paper we will give a survey of investigations on related results. This will be useful at the end of the paper to derive the above mentioned dependencies between the operators under study.
\end{abstract}

\section{INTRODUCTION}

Although being obviously more general than the standard Lebesgue spaces, variable exponent Lebesgue spaces preserve many of the properties of standard Lebesgue spaces. The most standard properties of variable exponent Lebesgue spaces may be seen in [12]. Namely, they are Banach spaces [12, Theorem 2.5], the Hölder inequality holds [12, Theorem 2.1], it is known a characterization for them to be reflexive [12, Corollary 2.7], and it is also known a condition under which continuous functions are dense [12, Theorem 2.11]. Anyway, variable exponent Lebesgue spaces appeared in the literature for the first time already in a 1931

Date: Received: Jan. 26, 2014; Accepted: May 1, 2014.

* Corresponding author.

2010 Mathematics Subject Classification. Primary 47B35; Secondary 47A53, 47A05, 47A20, $42 \mathrm{~A} 75$.

Key words and phrases. Wiener-Hopf operator, Hankel operator, almost periodic function, Fredholm property, invertibility. 
article by W. Orlicz [18]. This allowed the consideration of the so-called modular spaces which were systematically studied by H. Nakano [15, 16].

Following the development in variable exponent Lebesgue spaces (see the monographs [8, 12]), the research on operators defined between these spaces have recently increased significantly (cf., e.g., [6, 7]). Obviously, this facilitates the possibility to better understand applied problems e.g. in elasticity, fluid dynamics, calculus of variations and differential equations (cf., e.g., [19] for specific applications in electrorheological fluids). One of the difficulties in the development of the operator theory in the $L^{p(\cdot)}$ spaces is that the convolution operators are, in general, not bounded in these spaces. Some invertibility properties of Wiener-Hopf and Hankel operators - being well-known for standard Lebesgue spaces (cf., e.g., $[1,3,4,17])$ - are nowadays interestingly analysed in the framework of variable exponent Lebesgue spaces.

In the present paper, we will be devoted to analyse consequences and dependencies of the Fredholm and invertibility properties of Wiener-Hopf, Wiener-Hopf plus Hankel and Wiener-Hopf minus Hankel operators (in short, Wiener-HopfHankel operators) on variable exponent Lebesgue spaces and also on standard Lebesgue spaces.

In view to define these operators in a formal way, we will start by the definition of the variable exponent Lebesgue spaces. Let $p: \mathbb{R} \rightarrow[1, \infty]$ be measurable a.e. finite function. We denote by $L^{p(\cdot)}(\mathbb{R})$ the set of all complex-valued functions $f$ on $\mathbb{R}$ such that

$$
I_{p(\cdot)}\left(\frac{f}{\lambda}\right):=\int_{\mathbb{R}}\left|\frac{f(x)}{\lambda}\right|^{p(x)} d x<\infty
$$

for some $\lambda>0$. This set becomes a Banach space when equipped with the norm

$$
\|f\|_{p(\cdot)}:=\inf \left\{\lambda>0: I_{p(\cdot)}(f / \lambda) \leq 1\right\}
$$

The space $L^{p(\cdot)}(\mathbb{R})$ is precisely what we are refereing as the variable exponent Lebesgue space.

We will suppose that

$$
1<p_{-}:=\operatorname{ess}_{x \in \mathbb{R}} \inf p(x) \leq \operatorname{ess}_{x \in \mathbb{R}} \sup p(x)=: p_{+}<\infty .
$$

Under these conditions, the space $L^{p(\cdot)}(\mathbb{R})$ is separable and reflexive, and its dual space is isomorphic to $L^{p^{\prime}(\cdot)}(\mathbb{R})$, where $p^{\prime}(\cdot)$ is the conjugate exponent function defined by

$$
\frac{1}{p(x)}+\frac{1}{p^{\prime}(x)}=1 \quad(x \in \mathbb{R}) .
$$

Additionally, with condition (1.1) we have that $\|\phi I\|_{\mathcal{L}\left(L^{p(\cdot)}(\mathbb{R})\right)} \leq\|\phi\|_{L^{\infty}(\mathbb{R})}$ for a function $\phi \in L^{\infty}(\mathbb{R})$.

Moreover, $L^{p(\cdot)}\left(\mathbb{R}_{+}\right)$denotes the variable exponent Lebesgue space of complexvalued functions on the positive half-line $\mathbb{R}_{+}=(0,+\infty)$, and the subspace of $L^{p(\cdot)}(\mathbb{R})$ formed by all functions supported in the closure of $\mathbb{R}_{+}$is being denoted by $L_{+}^{p(\cdot)}(\mathbb{R})$. 
Given a Banach space $X$, we denote by $X^{N}$ the Banach space of all columns of height $N$ with components in $X$. The norm in $X^{N}$ is defined by

$$
\left\|\left(x_{1}, \ldots, x_{N}\right)^{\top}\right\|_{X^{N}}=\left(\sum_{j=1}^{N}\left\|x_{j}\right\|_{X}^{2}\right)^{1 / 2} .
$$

Given a subalgebra $B$ of $L^{\infty}(\mathbb{R})$, we denote by $B^{N \times N}$ the algebra of all $N \times N$ matrices $\Phi$ with entries in $B$. Moreover, we equip $B^{N \times N}$ with the norm

$$
\|\Phi\|_{B^{N \times N}}=\left\|\left(\Phi_{j k}\right)_{j, k=1}^{N}\right\|_{B^{N \times N}}=\left(\sum_{j, k=1}^{N}\left\|\Phi_{j k}\right\|_{B}^{2}\right)^{1 / 2} .
$$

We are now in condition to identify in a mathematical way the main objects of this work. We will consider Wiener-Hopf plus and minus Hankel operators acting between Lebesgue spaces with variable exponent $p(\cdot)$ (and also with fixed standard exponent $p(\cdot)=p \in(1,+\infty))$, denoted by

$$
W_{\Phi} \pm H_{\Phi}:\left[L_{+}^{p(\cdot)}(\mathbb{R})\right]^{N} \rightarrow\left[L^{p(\cdot)}\left(\mathbb{R}_{+}\right)\right]^{N},
$$

with $W_{\Phi}$ and $H_{\Phi}$ being Wiener-Hopf and Hankel operators defined by

$$
W_{\Phi}=r_{+} \mathcal{F}^{-1} \Phi \cdot \mathcal{F}, \quad H_{\Phi}=r_{+} \mathcal{F}^{-1} \Phi \cdot \mathcal{F} J
$$

respectively. Here, $r_{+}$represents the operator of restriction from $\left[L^{p(\cdot)}(\mathbb{R})\right]^{N}$ into $\left[L^{p(\cdot)}\left(\mathbb{R}_{+}\right)\right]^{N}, \mathcal{F}^{-1}$ denotes the inverse of the Fourier transformation $\mathcal{F}, \Phi$ is the socalled Fourier symbol, and $J:\left[L_{+}^{p(\cdot)}(\mathbb{R})\right]^{N} \rightarrow\left[L^{p(\cdot)}(\mathbb{R})\right]^{N}$ is the reflection operator given by the rule $J \varphi(x)=\widetilde{\varphi}(x)=\varphi(-x)$ which throughout the paper will be always considered for even functions $p(\cdot)$ (so that $J$ will therefore be a bounded operator in those variable exponent Lebesgue spaces).

One of the goals of this work is to exhibit conditions under which Wiener-Hopf plus and minus Hankel operators are both invertible in the full scale of standard Lebesgue spaces, in the case when the Fourier symbol $\Phi$ belongs to the so-called Wiener subclass of the almost periodic matrix functions (which we will define later on in detail).

\section{Preliminaries}

The boundedness of a wide variety of operators (and in particular of WienerHopf and Hankel operators) follows from the boundedness of the maximal operator on variable exponent Lebesgue spaces.

Given $f \in L_{\text {loc }}^{1}(\mathbb{R})$, the Hardy-Littlewood maximal operator $M$ is defined by

$$
(M f)(x):=\sup _{x \in \Omega} \frac{1}{|\Omega|} \int_{\Omega}|f(y)| d y,
$$

where the supremum is taken over all intervals $\Omega \subset \mathbb{R}$ containing $x$, and the Cauchy singular integral operator $S$ is defined by

$$
(S f)(x):=\frac{1}{\pi i} \int_{\mathbb{R}} \frac{f(\tau)}{\tau-x} d \tau
$$

where the integral is understood in the principal value sense. 
Theorem 2.1. (cf. e.g. [9, Theorem 2.1.]) Let $p: \mathbb{R} \rightarrow[1, \infty]$ be a measurable function satisfying (1.1). If the Hardy-Littlewood maximal operator $M$ is bounded on $L^{p(\cdot)}(\mathbb{R})$, then the Cauchy singular integral operator $S$ is bounded on $L^{p(\cdot)}(\mathbb{R})$.

The following result states a sufficient condition on $p(\cdot)$ for $M$ to be bounded on $L^{p(\cdot)}(\mathbb{R})$.

Theorem 2.2. (cf. e.g. [14, Theorem 2.5.]) Let $p: \mathbb{R} \rightarrow[1, \infty]$ satisfy (1.1). In addition, suppose that $p(\cdot)$ satisfies

$$
|p(x)-p(y)| \leq \frac{C}{-\log |x-y|}, \quad|x-y| \leq \frac{1}{2},
$$

and

$$
|p(x)-p(y)| \leq \frac{C}{\log (e+|x|)}, \quad|x| \leq|y| .
$$

Then, the Hardy-Littlewood maximal operator is bounded on $L^{p(\cdot)}(\mathbb{R})$.

Let $\mathcal{P}(\mathbb{R})$ denote the class of exponents $p: \mathbb{R} \rightarrow[1, \infty]$ continuous on $\mathbb{R}$ satisfying $(1.1)$, and let $\mathcal{B}(\mathbb{R})$ denote the set of all $p(\cdot) \in \mathcal{P}(\mathbb{R})$ such that $M$ is bounded on $L^{p(\cdot)}(\mathbb{R})$.

A function $\phi \in L^{\infty}(\mathbb{R})(p(\cdot) \in \mathcal{B}(\mathbb{R}))$ is called a Fourier multiplier on $L^{p(\cdot)}(\mathbb{R})$ if the operator $\mathcal{F}^{-1} \phi \mathcal{F}$ acting on $L^{2}(\mathbb{R}) \cap L^{p(\cdot)}(\mathbb{R})$, extends uniquely to a bounded operator on $L^{p(\cdot)}(\mathbb{R})$. The set of all Fourier multipliers on $L^{p(\cdot)}(\mathbb{R})$ is denoted by $\mathcal{M}_{p(\cdot)}$.

It follows that for $\Phi \in\left[\mathcal{M}_{p(\cdot)}\right]^{N \times N}$ (with an even $p(\cdot) \in \mathcal{B}(\mathbb{R})$ ) the Wiener-Hopf and the Hankel operators defined in $(1.2), W_{\Phi}:\left[L_{+}^{p(\cdot)}(\mathbb{R})\right]^{N} \rightarrow\left[L^{p(\cdot)}\left(\mathbb{R}_{+}\right)\right]^{N}$ and $H_{\Phi}:\left[L_{+}^{p(\cdot)}(\mathbb{R})\right]^{N} \rightarrow\left[L^{p(\cdot)}\left(\mathbb{R}_{+}\right)\right]^{N}$, are bounded. These are in fact necessary and sufficient conditions for the Wiener-Hopf plus and minus Hankel operators to be bounded in variable exponent Lebesgue spaces. In a sense, these conditions are not explicit. For some more explicit conditions which determine the identification of subsets of $\mathcal{M}_{p(\cdot)}$, we would like to refer the reader to $[10, \S 4.2]$ where, in particular, a generalization of the classical Mikhlin's theorem on Fourier multipliers (cf. [13]) to variable exponent Lebesgue spaces is presented.

In what follows, we will be also using the projection $P$ and its complementary projection $Q(Q=I-P)$, where

$$
P=\mathcal{F} \ell_{0} r_{+} \mathcal{F}^{-1},
$$

with $\ell_{0}:\left[L^{p(\cdot)}\left(\mathbb{R}_{+}\right)\right]^{N} \rightarrow\left[L_{+}^{p(\cdot)}(\mathbb{R})\right]^{N}$ being the zero extension operator.

Let $\dot{\mathbb{R}}:=\mathbb{R} \cup\{\infty\}$. We will denote by $C(\dot{\mathbb{R}})$ the set of all continuous functions $\varphi$ on the real line for which the two limits

$$
\varphi(-\infty):=\lim _{x \rightarrow-\infty} \varphi(x), \quad \varphi(+\infty):=\lim _{x \rightarrow+\infty} \varphi(x)
$$

exist and coincide. The common value of these two limits will be denoted by $\varphi(\infty)$. Furthermore, $C_{0}(\dot{\mathbb{R}})$ will represent the collection of all $\varphi \in C(\dot{\mathbb{R}})$ for which $\varphi(\infty)=0$. 
For a continuous function $f: \mathbb{R} \rightarrow \mathbb{C}$ and $\mathcal{C} \subset \mathbb{R}$, let

$$
\operatorname{osc}(f, \mathcal{C}):=\sup _{t, \tau \in \mathcal{C}}|f(t)-f(\tau)| .
$$

We denote by $S O$ the class of slowly oscillating functions given by

$$
S O:=\left\{f \in C(\mathbb{R}): \lim _{x \rightarrow+\infty} \operatorname{osc}(f,[-2 x .-x] \cup[x, 2 x])=0\right\} \cap L^{\infty}(\mathbb{R}) .
$$

$S O$ is a unital $C^{*}$-algebra of $L^{\infty}(\mathbb{R})$ that contains $C(\dot{\mathbb{R}})$.

In what follows, we will use the notation $\mathcal{G} B$ for the group of all invertible elements of a Banach algebra $B$.

\section{Relations BetWeEn DifFERENT Classes of CONVOLUtion tyPE OPERATORS}

In order to relate operators and to transfer certain operator properties between different types of operators, we will also use the notion of equivalence relation and equivalence after extension relation between bounded linear operators. We will now recall these concepts in detail.

Consider two bounded linear operators $T: X_{1} \rightarrow X_{2}$ and $S: Y_{1} \rightarrow Y_{2}$, acting between Banach spaces. The operators $T$ and $S$ are said to be equivalent if there are two boundedly invertible linear operators, $E: Y_{2} \rightarrow X_{2}$ and $F: X_{1} \rightarrow Y_{1}$, such that

$$
T=E S F .
$$

It directly follows from (3.1) that if two operators are equivalent, then they belong to the same invertibility class. More precisely, one of these operators is invertible, left invertible, right invertible or only generalized invertible, if and only if the other operator enjoys the same property.

We say that $T$ is equivalent after extension to $S$ (and write $T \stackrel{*}{\sim} S$ ) if there are Banach spaces $Z_{1}$ and $Z_{2}$ and invertible bounded linear operators $E$ and $F$ such that

$$
\left[\begin{array}{cc}
T & 0 \\
0 & I_{Z_{1}}
\end{array}\right]=E\left[\begin{array}{cc}
S & 0 \\
0 & I_{Z_{2}}
\end{array}\right] F
$$

where $I_{Z_{1}}$ and $I_{Z_{2}}$ represent the identity operators in $Z_{1}$ and $Z_{2}$, respectively. In case that $Z_{1}$ or $Z_{2}$ are the trivial space, we will say that the operators $T$ and $S$ are equivalent after one-sided extension.

If two operators are equivalent after extension, then they belong to the same invertibility class.

The following theorem is well-known (cf. [2]) when considering $L^{\infty}$ Fourier symbols in Wiener-Hopf and Hankel operators acting between standard $L^{p}$-spaces. Here, we present its generalization by including the variable exponent Lebesgue spaces case.

Theorem 3.1. Let $\Phi \in \mathcal{G}\left[\mathcal{M}_{p(\cdot)}\right]^{N \times N}$ with an even $p(\cdot) \in \mathcal{B}(\mathbb{R})$ in the case of variable exponent Lebesgue spaces or simply $\Phi \in \mathcal{G}\left[\mathcal{M}_{p}\right]^{N \times N}$ with $p(\cdot)=p \in$ $(1,+\infty)$ in the case of standard Lebesgue spaces. The following two operators are equivalent after extension: 
(i) $\left[\begin{array}{cc}W_{\Phi}+H_{\Phi} & 0 \\ 0 & W_{\Phi}-H_{\phi}\end{array}\right]:\left[L_{+}^{p(\cdot)}(\mathbb{R})\right]^{2 N} \rightarrow\left[L^{p(\cdot)}\left(\mathbb{R}_{+}\right)\right]^{2 N} ;$
(ii) $W_{\Phi \widetilde{\Phi^{-1}}}:\left[L_{+}^{p(\cdot)}(\mathbb{R})\right]^{N} \rightarrow\left[L^{p(\cdot)}\left(\mathbb{R}_{+}\right)\right]^{N}$.

Proof. We start by noticing that the operators

$$
W_{\Phi} \pm H_{\Phi}:\left[L_{+}^{p(\cdot)}(\mathbb{R})\right]^{N} \rightarrow\left[L^{p(\cdot)}\left(\mathbb{R}_{+}\right)\right]^{N}
$$

and

$$
P \phi P \pm P \phi J P+Q:\left[L^{p(\cdot)}(\mathbb{R})\right]^{N} \rightarrow\left[L^{p(\cdot)}(\mathbb{R})\right]^{N}
$$

are equivalent after extension. Indeed, due to the identity

$$
P \Phi P \pm P \Phi J P+Q=(\Phi P \pm \Phi J P+Q)(I-Q \Phi P \mp Q \Phi J P),
$$

where the operator $I-Q \Phi P \mp Q \Phi J P$ is invertible (with inverse being given by $I+Q \Phi P \mp Q \Phi J P)$, it follows that the operator in (i) is equivalent after extension to the operator

$$
\left[\begin{array}{cc}
\Phi P+\Phi J P+Q & 0 \\
0 & \Phi P-\Phi J P+Q
\end{array}\right]:\left[L^{p(\cdot)}(\mathbb{R})\right]^{2 N} \rightarrow\left[L^{p(\cdot)}(\mathbb{R})\right]^{2 N} .
$$

Observing that

$$
\begin{array}{r}
\frac{1}{2}\left[\begin{array}{cc}
I & I \\
J & -J
\end{array}\right]\left[\begin{array}{cc}
\Phi P+\Phi J P+Q & 0 \\
0 & \Phi P-\Phi J P+Q
\end{array}\right]\left[\begin{array}{cc}
I & J \\
I & -J
\end{array}\right] \\
=\left[\begin{array}{cc}
\Phi & 0 \\
\widetilde{\Phi} & I
\end{array}\right] P+\left[\begin{array}{cc}
I & \Phi \\
0 & \widetilde{\Phi}
\end{array}\right] Q,
\end{array}
$$

and multiplying this operator from the left with the invertible operator

$$
A:=\left[\begin{array}{cc}
I & -\widetilde{\Phi^{-1}} I \\
0 & \widetilde{\Phi^{-1} I}
\end{array}\right]
$$

we obtain the equivalent operator $\Psi P+Q$, where

$$
\Psi=\left[\begin{array}{cc}
0 & -\widetilde{\Phi \Phi^{-1}} \\
1 & \widetilde{\Phi^{-1}}
\end{array}\right]
$$

Since

$$
\Psi P+Q=(P \Psi P+Q)(I+Q \Psi P),
$$

where the operator on the right-hand side of (3.2) is invertible (with inverse being given by $I-Q \Psi P$ ), it follows that the operator in (ii) is equivalent to the operator $P \Psi P+Q$ which in its turn is equivalent after extension to the operator $P \Psi P$.

Additionally,

$$
P\left[\begin{array}{cc}
0 & -\widetilde{\Phi \Phi^{-1}} \\
1 & \widetilde{\Phi^{-1}}
\end{array}\right] P=P\left[\begin{array}{cc}
\widetilde{\Phi \Phi^{-1}} & 0 \\
0 & I
\end{array}\right] P\left[\begin{array}{cc}
0 & -I \\
I & \widetilde{\Phi^{-1}}
\end{array}\right] P .
$$


Since

$$
B:=P\left[\begin{array}{cc}
0 & -I \\
I & \widetilde{\Phi^{-1}}
\end{array}\right] P
$$

is invertible, (3.3) shows an explicit equivalence after (one-sided) extension between $P \Psi P$ and $P \Phi \widetilde{\Phi^{-1}} P$. Due to the fact that $P \Phi \widetilde{\Phi^{-1} P}$ is equivalent to $W_{\Phi \widetilde{\Phi^{-1}}}$, altogether, it follows that the operators in (i) and (ii) are equivalent after extension.

Finally, for the case of variable exponent Lebesgue spaces, we stress that all the just used operators are bounded in the variable exponent Lebesgue spaces due to the hypothesis $\Phi \in \mathcal{G}\left[\mathcal{M}_{p(\cdot)}\right]^{N \times N}$ and the consideration of an even $p(\cdot) \in$ $\mathcal{B}(\mathbb{R})$.

\section{Matrix-VAlued $A P$ Symbols}

The smallest closed subalgebra of $L^{\infty}(\mathbb{R})$ that contains all the functions $e_{\lambda}(\lambda \in$ $\mathbb{R})$, where $e_{\lambda}(x)=e^{i \lambda x}, x \in \mathbb{R}$, is denoted by $A P$ and called the algebra of almost periodic functions:

$$
A P:=\operatorname{alg}_{L^{\infty}(\mathbb{R})}\left\{e_{\lambda}: \lambda \in \mathbb{R}\right\} .
$$

In addition, we use the notation

$$
A P_{+}:=\operatorname{alg}_{L^{\infty}(\mathbb{R})}\left\{e_{\lambda}: \lambda \geq 0\right\}, \quad A P_{-}:=\operatorname{alg}_{L^{\infty}(\mathbb{R})}\left\{e_{\lambda}: \lambda \leq 0\right\}
$$

for these two subclasses of $A P$ (which are still closed subalgebras of $L^{\infty}(\mathbb{R})$ ).

We will likewise make use of the Wiener subclass of $A P$ (denoted by $A P W$ ) formed by all those elements from $A P$ which allow a representation by an absolutely convergent series. Therefore, $A P W$ is precisely the (proper) subclass of all functions $\varphi \in A P$ which can be written in an absolutely convergent series of the form:

$$
\varphi=\sum_{j} \varphi_{j} e_{\lambda_{j}}
$$

where $\lambda_{j} \in \mathbb{R}$ and $\sum_{j}\left|\varphi_{j}\right|<\infty$.

We recall that all $A P$ functions have a well-known mean value. The existence of such a number is provided in the next standard result from the theory of almost periodic functions (cf., e.g., [5]).

Proposition 4.1. Let $A \subset(0, \infty)$ be an unbounded set and let

$$
\left\{I_{\alpha}\right\}_{\alpha \in A}=\left\{\left(x_{\alpha}, y_{\alpha}\right)\right\}_{\alpha \in A}
$$

be a family of intervals $I_{\alpha} \subset \mathbb{R}$ such that $\left|I_{\alpha}\right|=y_{\alpha}-x_{\alpha} \rightarrow \infty$ as $\alpha \rightarrow \infty$. If $\varphi \in A P$, then the limit

$$
M(\varphi):=\lim _{\alpha \rightarrow \infty} \frac{1}{\left|I_{\alpha}\right|} \int_{I_{\alpha}} \varphi(x) d x
$$

exists, is finite, and is independent of the particular choice of the family $\left\{I_{\alpha}\right\}$. 
For any $\varphi \in A P$, the number that has been just introduced $M(\varphi)$ is called the Bohr mean value or simply the mean value of $\varphi$. In the matrix case the mean value is defined entry-wise.

Let $A P W^{-}\left(A P W^{+}\right)$be the set of all functions $\psi \in A P W$ such that $\Omega(\psi) \subset$ $(-\infty, 0]\left(\Omega(\psi) \subset[0,+\infty)\right.$, respectively). Here $\Omega(\psi)=\left\{\lambda \in \mathbb{R}: M\left(\psi e_{-\lambda}\right) \neq 0\right\}$ is the Bohr-Fourier spectrum of $\psi$. Naturally, $A P W^{-} \subset A P^{-}$and $A P W^{+} \subset A P^{+}$.

Let us now recall the so-called right $A P$ and $A P W$ factorizations and some other notions associated with them.

Definition 4.2. A matrix function $\Phi \in \mathcal{G} A P^{N \times N}$ is said to admit a right $A P$ factorization if it can be represented in the form

$$
\Phi(x)=\Phi_{-}(x) D(x) \Phi_{+}(x)
$$

for all $x \in \mathbb{R}$, with

$$
\Phi_{-} \in \mathcal{G} A P_{-}^{N \times N}, \quad \Phi_{+} \in \mathcal{G} A P_{+}^{N \times N},
$$

and $D$ is a diagonal matrix of the form $D(x)=\operatorname{diag}\left[e^{i \lambda_{1} x}, \ldots, e^{i \lambda_{N} x}\right], \lambda_{j} \in \mathbb{R}$. The numbers $\lambda_{j}$ are called the right $A P$ indices of the factorization. A right $A P$ factorization with $D=I_{N \times N}$ is referred to be a canonical right $A P$ factorization. In the case when (4.1) is substituted by

$$
\Phi_{-} \in \mathcal{G} A P W_{-}^{N \times N}, \Phi_{+} \in \mathcal{G} A P W_{+}^{N \times N},
$$

we will be in the presence of a right $A P W$ factorization which will also turn to be a canonical one in case of $D=I_{N \times N}$.

The vector containing the right $A P$ indices will be denoted by $k(\Phi)$, i.e., in the above case $k(\Phi):=\left(\lambda_{1}, \ldots, \lambda_{N}\right)$. If we consider the case with equal right $A P$ indices $\left(k(\Phi):=\left(\lambda_{1}, \lambda_{1}, \ldots, \lambda_{1}\right)\right)$, then the matrix $\mathbf{d}(\Phi):=M\left(\Phi_{-}\right) M\left(\Phi_{+}\right)$is independent of the particular choice of the right $A P$ factorization. In this case, this matrix $\mathbf{d}(\Phi)$ is called the geometric mean of $\Phi$.

The just presented factorizations allow the possibility to find conditions which induce the invertibility or, at least, the Fredholm property of Wiener-Hopf operators in standard Lebesgue spaces. Anyway, for $A P W^{N \times N}$ even stronger results appear as it is the case of the following Kurbatov Theorem (cf. [11]).

Theorem 4.3. If $\Phi \in A P W^{N \times N}$, then $W_{\Phi}:\left[L_{+}^{p}(\mathbb{R})\right]^{N} \rightarrow\left[L^{p}\left(\mathbb{R}_{+}\right)\right]^{N}$ is invertible for some $p \in(1,+\infty)$ if and only if $W_{\Phi}:\left[L_{+}^{p}(\mathbb{R})\right]^{N} \rightarrow\left[L^{p}\left(\mathbb{R}_{+}\right)\right]^{N}$ is invertible for all $p \in(1,+\infty)$.

\section{MATRIX-VAlued $S A P$ Symbols}

The $C^{*}$-algebra $S A P$ of all semi-almost periodic functions on $\mathbb{R}$ is the smallest closed subalgebra of $L^{\infty}(\mathbb{R})$ that contains $A P$ and $C(\overline{\mathbb{R}})$ :

$$
S A P=\operatorname{alg}_{L^{\infty}(\mathbb{R})}\{A P, C(\overline{\mathbb{R}})\} .
$$

Here, we denote by $C(\overline{\mathbb{R}})$ the set of all continuous functions on $\mathbb{R}$ with a possible jump at infinity. In addition, it is possible to interpret the $S A P$ functions in a different form due to the following characterization of D. Sarason [20]. 
Theorem 5.1. Let $u \in C(\overline{\mathbb{R}})$ be any function for which $u(-\infty)=0$ and $u(+\infty)=$ 1. If $\varphi \in S A P$, then there is $\varphi_{\ell}, \varphi_{r} \in A P$ and $\varphi_{0} \in C_{0}(\dot{\mathbb{R}})$ such that

$$
\varphi=(1-u) \varphi_{\ell}+u \varphi_{r}+\varphi_{0} .
$$

The functions $\varphi_{\ell}, \varphi_{r}$ are uniquely determined by $\varphi$, and independent of the particular choice of $u$. The maps

$$
\varphi \mapsto \varphi_{\ell}, \quad \varphi \mapsto \varphi_{r}
$$

are $C^{*}$-algebra homomorphisms of $S A P$ onto $A P$.

The last theorem is also valid in the matrix case. In the next section we will use the following result which allows a certain connection between the Fredholm property of some classes of singular integral operators on variable exponent Lebesgue spaces and consequent invertible operators on specific standard Lebesgue spaces.

Theorem 5.2. ([9, Theorem 1.2]) Let $\Phi \in S A P^{N \times N}$ and $p(\cdot) \in \mathcal{B}(\mathbb{R}) \cap S O$. If the singular integral operator $\Phi P+Q$ is Fredholm on the variable Lebesgue space $\left[L^{p(\cdot)}(\mathbb{R})\right]^{N}$, then:

(a) there is an exponent $q_{r}$ lying in the segment

$$
\left[\lim _{x \rightarrow+\infty} \inf p(x), \lim _{x \rightarrow+\infty} \sup p(x)\right]
$$

such that $\Phi_{r} P+Q$ is invertible on the standard Lebesgue space $\left[L^{q_{r}}(\mathbb{R})\right]^{N}$;

(b) there is an exponent $q_{\ell}$ lying in the segment

$$
\left[\lim _{x \rightarrow-\infty} \inf p(x), \lim _{x \rightarrow-\infty} \sup p(x)\right]
$$

such that $\Phi_{\ell} P+Q$ is invertible on the standard Lebesgue space $\left[L^{q \ell}(\mathbb{R})\right]^{N}$.

\section{Wiener-Hopf And Wiener-Hopf-Hankel operators With $A P W$ FOURIER SYMBOLS IN DIFFERENT LEBESGUE SPACES}

Making use of the previous results and, in particular, the equivalence after extension relation presented in Theorem 3.1, we are in position to describe an interplay between the Fredholm property of a Wiener-Hopf operator acting on variable exponent Lebesgue spaces and the invertibility of some associated Wiener-Hopf plus and minus Hankel operators on standard Lebesgue spaces in all the possible range of the $p$ Lebesgue constant exponents.

Theorem 6.1. Let $\Phi \in \mathcal{G} A P W^{N \times N}$ and $p(\cdot) \in \mathcal{B}(\mathbb{R}) \cap S O$. If the Wiener-Hopf operator acting between variable exponent Lebesgue spaces

$$
W_{\Phi \widetilde{\Phi^{-1}}}:\left[L_{+}^{p(\cdot)}(\mathbb{R})\right]^{N} \rightarrow\left[L^{p(\cdot)}\left(\mathbb{R}_{+}\right)\right]^{N}
$$

has the Fredholm property, then the Wiener-Hopf plus Hankel and the WienerHopf minus Hankel operators

$$
W_{\Phi} \pm H_{\Phi}:\left[L_{+}^{p}(\mathbb{R})\right]^{N} \rightarrow\left[L^{p}\left(\mathbb{R}_{+}\right)\right]^{N}
$$

are both invertible operators for all $p \in(1,+\infty)$. 
Proof. Having in mind that $\Phi \in \mathcal{G} A P W^{N \times N}$, we also have $\Phi \widetilde{\Phi \Phi^{-1}} \in A P W^{N \times N}$ and, moreover,

$$
\left(\Phi \widetilde{\Phi^{-1}}\right)_{\ell}=\left(\widetilde{\Phi \Phi^{-1}}\right)_{r}=\Phi \widetilde{\Phi^{-1}}
$$

Therefore, from Theorem 5.2, we obtain that there is an exponent

$$
q \in\left[p_{-}, p_{+}\right]
$$

(cf. (1.1)) such that the singular integral operator $\Phi \widetilde{\Phi-1} P+Q$ is invertible on the standard Lebesgue space $\left[L^{q}(\mathbb{R})\right]^{N}$.

However,

$$
\Phi \widetilde{\Phi^{-1}} P+Q:\left[L^{q}(\mathbb{R})\right]^{N} \rightarrow\left[L^{q}(\mathbb{R})\right]^{N}
$$

is equivalent after extension to $W_{\Phi \widetilde{\Phi^{-1}}}:\left[L_{+}^{q}(\mathbb{R})\right]^{N} \rightarrow\left[L^{q}\left(\mathbb{R}_{+}\right)\right]^{N}$. Thus, $W_{\Phi \widetilde{\Phi^{-1}}}$ is also invertible on the standard Lebesgue space $\left[L_{+}^{q}(\mathbb{R})\right]^{N}$.

Therefore, due to the Kurbatov Theorem,

$$
W_{\Phi \widetilde{\Phi^{-1}}}:\left[L_{+}^{p}(\mathbb{R})\right]^{N} \rightarrow\left[L^{p}\left(\mathbb{R}_{+}\right)\right]^{N}
$$

is an invertible operator for all $p \in(1,+\infty)$. Using the equivalence after extension relation, presented in Theorem 3.1, we obtain that the Wiener-Hopf plus and minus Hankel operators $W_{\Phi}+H_{\Phi}:\left[L_{+}^{p}(\mathbb{R})\right]^{N} \rightarrow\left[L^{p}\left(\mathbb{R}_{+}\right)\right]^{N}$ and $W_{\Phi}-H_{\Phi}$ : $\left[L_{+}^{p}(\mathbb{R})\right]^{N} \rightarrow\left[L^{p}\left(\mathbb{R}_{+}\right)\right]^{N}$ are both invertible operators for all $p \in(1,+\infty)$.

Acknowledgement. This work was supported in part by Portuguese funds through the CIDMA - Center for Research and Development in Mathematics and Applications, and the Portuguese Foundation for Science and Technology ("FCT-Fundação para a Ciência e a Tecnologia"), within project PEst-OE/MAT/ UI4106/2014.

\section{REFERENCES}

1. G. Bogveradze and L.P. Castro, Invertibility characterization of Wiener-Hopf plus Hankel operators via odd asymmetric factorizations, Banach J. Math. Anal. 3 (2009), no. 1, 1-18.

2. L.P. Castro and A.S. Silva, Invertibility of matrix Wiener-Hopf plus Hankel operators with symbols producing a positive numerical range, Z. Anal. Anwend. 28 (2009), no. 1, 119-127.

3. L.P. Castro and A.S Silva, Invertibility criteria for Wiener-Hopf plus Hankel operators with different almost periodic Fourier symbol matrices, Ann. Mat. Pura Appl. 192 (2013), 1141-1152.

4. L.P. Castro and F.-O. Speck, Inversion of matrix convolution type operators with symmetry, Port. Math. 62 (2005), 193-216.

5. C. Corduneanu, Almost Periodic Functions, J. Wiley \& Sons, New York, 1968.

6. D. Cruz-Uribe, A. Fiorenza, J.M. Martell and C. Pérez, The boundedness of classical operators on variable $L^{p}$ spaces, Ann. Acad. Sci. Fenn. Math. 31 (2006), no. 1, 239-264.

7. L. Diening, Maximal function on Musielak-Orlicz spaces and generalized Lebesgue spaces, Bull. Sci. Math. 129 (2005), 657-700.

8. L. Diening, P. Harjulehto, P. Hästö and M. Rư̌ičcka, Lebesgue and Sobolev Spaces with Variable Exponents, Lecture Notes in Math., 2017, Springer, Heidelberg, 2011.

9. A. Yu. Karlovich and I.M. Spitkovsky, On singular integral operators with semi-almost periodic coefficients on variable Lebesgue spaces, J. Math. Anal. Appl. 384 (2011), no. 2, $706-725$.

10. V.M. Kokilashvili and S.G. Samko, Operators of harmonic analysis in weighted spaces with non-standard growth, J. Math. Anal. Appl. 352 (2009), no. 1, 15-34. 
11. V.G. Kurbatov, Functional-Differential Operators and Equations, Kluwer Academic Publishers, Dordrecht, 1999.

12. O. Kováčik and J. Rákosník, On spaces $L^{p(x)}$ and $W^{k, p(x)}$, Czechoslovak Math. J. 41 (1991), no. 4, 592-618.

13. S.G. Mikhlin, On multipliers of Fourier integrals (in Russian), Dokl. Akad. Nauk SSSR 109 (1956), 701-703.

14. J. Motos, M.J. Planells and C.F. Talavera, On variable exponent Lebesgue spaces of entire analytic functions, J. Math. Anal. Appl. 388 (2012), 775-787.

15. H. Nakano, Modulared Semi-Ordered Linear Spaces, Maruzen Co. Ltd., Tokyo, 1950.

16. H. Nakano, Topology of Linear Topological Spaces, Maruzen Co. Ltd., Tokyo, 1951.

17. A.P. Nolasco and L.P. Castro, A Duduchava-Saginashvili's type theory for Wiener-Hopf plus Hankel operators, J. Math. Anal. Appl. 331 (2007), 329-341.

18. W. Orlicz, Über konjugierte Exponentenfolgen, Studia Math. 3 (1931), 200-212.

19. M. Rǔzička, Electrorheological Fluids: Modeling and Mathematical Theory, Lecture Notes in Math., 1748, Springer, Berlin, 2000.

20. D. Sarason, Toeplitz operators with semi-almost periodic symbols, Duke Math. J. 44 (1977), $357-364$.

Cidma-Center for Research and Development in Mathematics and Applications, Department of Mathematics, University of Aveiro, 3810-193 Aveiro, PorTUGAL.

E-mail address: castro@ua.pt

E-mail address: anabela.silva@ua.pt 